\title{
¿Cómo elaborar correctamente preguntas de elección múltiple?
}

\author{
J. Palés-Argullós
}

\section{Introducción}

Los exámenes de preguntas de elección múltiple son uno de los instrumentos de tipo escrito más utilizados en la evaluación en educación médica -preguntas de respuesta múltiple (PRM) o multiple choice question (MCQ), en inglés). Tanto es así, que en muchos casos viene a ser el único instrumento utilizado en numerosos procesos de evaluación. Suelen denominarse 'pruebas objetivas' por su demostrada alta fiabilidad, aunque para ello deben estar bien diseñados. De hecho, confeccionarlos adecuadamente requiere un cierto nivel de experiencia.

Respecto a su validez, son adecuados para medir un espectro amplio del conocimiento y se pueden usar con seguridad para medir los componentes relacionados con los conocimientos y, hasta cierto punto, la capacidad de aplicarlos, siempre y cuando se formulen adecuadamente. Otras capacidades que pueden evaluarse con los exámenes de PRM son el razonamiento clínico y la toma de decisiones. Por otra parte, tienen otra ventaja y es que son fáciles de administrar y corregir, especialmente con números altos de alumnos.

Sin embargo, también se le han dedicado críticas porque existe poca semejanza entre los exámenes de este tipo y el trabajo de un clínico, y no son representativos del amplio abanico de actividades intelectuales que se asumen que deben demostrar los estudiantes de ciencias de la salud y profesionales sanitarios.

A pesar de su uso tan extensivo, no resulta infrecuente encontrarse con exámenes de este tipo con preguntas mal formuladas desde el punto de vista técnico. Este breve artículo pretende dar unas reglas generales para confeccionar correctamente este tipo de preguntas y señalar los defectos técnicos más frecuentemente observados con el fin de que puedan evitarse.

\section{Reglas generales}

En primer lugar, se debe señalar como norma general que el contenido del examen debe coincidir con los objetivos del curso y los temas fundamentales han de tener más peso que los temas menos importantes. Es muy recomendable por ello establecer una tabla de especificaciones y determinar con anterioridad cuántas preguntas de cada parte del curso o de la asignatura se van a elaborar, de acuerdo con su importancia y pertinencia.

Cada pregunta debe centrarse en un concepto importante, que normalmente será un problema clínico común o potencialmente grave, o un aspecto importante de las ciencias básicas. Se deben evitar preguntas que evalúen el conocimiento de datos triviales.

Por otra parte, siempre que sea posible hay que huir de confeccionar preguntas que sólo exploren la capacidad de recordar conocimientos abstractos. Si no lo hacemos, no sacaremos todo el provecho que nos brinda este tipo de preguntas. Pero si una misma pregunta la planteamos en un contexto, podrá servir además para evaluar si el candidato sabe cómo aplicar los conocimientos. Comparemos la pregunta del cuadro 1:

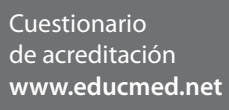

www.educmed.net

Facultad de Medicina,

Universitat de Barcelona,

Sociedad Española

de Educación Médica.

Fundación Educación

Médica.

Correspondencia

Dr. Jordi Palés Argullós.

Departamento de

Ciencias Fisiológicas I.

Facultad de Medicina.

Universitat de Barcelona.

Casanova, 143.

E-08036 Barcelona.

E-mail

jpales@ub.edu

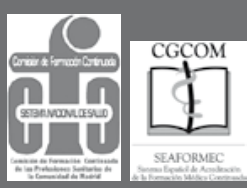

Actividad acreditada por SEAFORMEC con 1,5 créditos

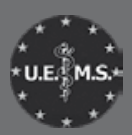

Créditos reconocidos por el 'European

Accreditation Council for CME' de la UEMS 
Cuadro 1 (tomado de [1]).

a) ¿Cuál de las siguientes situaciones es la más probable en un shock cardiogénico?

A. Hipoxemia con pH normal.

B. Acidosis metabólica.

C. Alcalosis metabólica.

D. Acidosis respiratoria

E. Alcalosis respiratoria

b) Enunciado propuesto con las mismas posibles opciones: Una mujer de 74 años ingresa en urgencias por un dolor torácico. Está inquieta, confusa y diaforética. Al ingreso, temperatura de $36,7^{\circ} \mathrm{C}$, tensión arterial de $148 / 78 \mathrm{mmHg}$ pulso de 90 latidos/min, y frecuencia respiratoria de 24 inspiraciones/min. Durante la siguiente hora, pasa a un estado de estupor, la tensión arterial disminuye a $80 / 40 \mathrm{mmHg}$ el pulso aumenta a 120 latidos/min y la frecuencia respiratoria es de 40 inspiraciones/min. Piel fría. El ECG muestra ritmo sinusal y un segmento ST de $4 \mathrm{~mm}$ elevado en las derivaciones V2 a V6. La gasometría arterial pondrá de manifiesto: (mismas opciones que en a).

En el fondo es la misma pregunta, pero en el caso $b$ se plantea en un determinado contexto que es el que refleja la viñeta. Esto es válido tanto para las preguntas clínicas como para las que pretenden evaluar las ciencias básicas (Cuadro 2):

Cuadro 2 (tomado de [1]).

a) ¿Qué región recibe el suministro sanguíneo a través de la arteria cerebelosa inferior posterior? A. ... B. ... C.... D. ... E. ...

b) Un hombre de 62 años de edad presenta ataxia de las extremidades del lado izquierdo, síndrome de Horner nistagmo y pérdida de las sensaciones de dolor y temperatura faciales. ¿Qué arteria es más probable que esté obstruida? A. ... B. ... C.... D. ... E. ...

Es recomendable por ello construir PRM en base a viñetas que determinen un contexto concreto, tanto en las ciencias clínicas como básicas. Para el caso de las ciencias clínicas, cada pregunta debería comenzar con la presentación del problema de un paciente. Luego, debería continuar con la historia clínica (que incluya la duración de los signos y síntomas), los datos del examen físico, resultados de los estudios de diagnóstico, tratamiento inicial, hallazgos posteriores, etc. Las viñetas pueden incluir solamente un subgrupo de esta información, pero se debería presentar la información en el orden especificado. En estos casos, el enunciado puede ser relativamente extenso, pero en cualquier caso las opciones deben ser breves. Para el área de las ciencias básicas, la viñeta del paciente o de una situación normal puede ser muy breve.

En cualquier caso, el enunciado de la pregunta debe generar una pregunta clara y debe ser posible llegar a la respuesta con las opciones ocultas. Para determinar si la pregunta está centrada en un tema en particular, se deben ocultar las opciones y observar si la pregunta es clara y si los alumnos pueden intentar responderla solamente a partir de la información en el enunciado. Si no es posible, vuelva a elaborar el contenido del enunciado o de las opciones.

Todos los distractores (es decir, las opciones incorrectas) deben ser homogéneos. Deben estar dentro de la misma categoría que la respuesta correcta (p. ej., diagnósticos, análisis, tratamientos, pronósticos, alternativas de disposición).

Todos los distractores deben ser convincentes, gramaticalmente correctos, lógicamente compatibles y de la misma extensión (relativa) que la respuesta correcta. Las opciones deben ordenarse de manera lógica (p. ej., numéricamente) o en orden alfabético. Se deben distribuir las opciones correctas entre las diversas opciones.

\section{Defectos técnicos a evitar}

Al elaborar las PRM es frecuente cometer errores técnicos que facilitan o dificultan la tarea del estudiante. Analicemos algunos de los más frecuentes y qué hay que evitar hacer. Se resumen en la tabla I.

No se debe:

- Formular enunciados que supongan juicios de valor u opiniones personales (debemos evitar enunciados del tipo: ‘ ¿cuál considera usted la conducta más adecuada?', ‘ ¿cuál piensa usted que es la opción correcta').

- Proponer opciones o distractores que no tengan correspondencia gramatical con el enunciado. Cuando esto ocurre, el estudiante tiende a descartarlas de entrada, con lo cual las posibilidades reales se reducen (Cuadro 3). Cada opción debe poderse leer directamente a continuación del enunciado. 


\section{Programa de Formación Médica Acreditada en Educación Médica}

Artículo: ¿Cómo elaborar correctamente preguntas de elección múltiple?

Autor: J. Palés-Argullós

Revista: Educ Med 2010; 13: 149-55
Encontrará este cuestionario en la web de la revista: www.educmed.net

Marque en cada caso los defectos técnicos que contengan las preguntas señaladas. La numeración de las preguntas corresponde a la que se señala en la tabla II. La numeración de los defectos corresponde a la que se señala en la tabla I. Cada pregunta puede tener más de un defecto.
1. La pregunta 1 contiene el/los defectos/s:
6. La pregunta 6 contiene el/los defecto/s:
$\square$ a) 1
$\square$ a) 1
$\square$ b) 2
$\square$ b) 2
$\square$ c) 3
$\square$ c) 3
$\square$ d) 4
$\square$ d) 4
$\square$ e) No contiene ningún defecto
$\square$ e) No contiene ningún defecto

2. La pregunta 2 contiene el/los defecto/s:

7. La pregunta 7 contiene el/los defecto/s:

$\square$ a) 6

$\square$ a) 4

$\square$ b) 7

$\square$ b) 6

ㄷ) 9

$\square$ c) 8

$\square$ d) 10

$\square$ d) 10

$\square$ e) 11

$\square$ e) 12

\section{La pregunta 3 contiene el/los defecto/s:}

8. La pregunta 8 contiene el/los defecto/s:

$\square$ a) 1

$\square$ b) 3

$\square$ a) 1

$\square$ c) 5

$\square$ b) 4

$\square$ d) 11

$\square$ c) 6

$\square$ e) No contiene ningún defecto

4. La pregunta 4 contiene el/los defecto/s:

$\square$ e) 10
$\square$ a) 4
$\square$ b) 6
$\square$ a) 2
$\square$ c) 8
$\square$ b) 6
$\square$ d) 10
$\square$ c) 8
$\square$ e) 12
$\square$ d) 10
$\square$ e) 12

9. La pregunta 9 contiene el/los defecto/s:

5. La pregunta 5 contiene el/los defecto/s:
$\square$ a) 1
$\square$ b) 3
$\square$ a) 1
$\square$ c) 5
$\square$ b) 3
$\square$ d) 7
$\square$ c) 5
$\square$ e) No contiene ningún defecto
$\square$ d) 7
$\square$ e) No contiene ningún defecto

10. La pregunta 10 contiene el/los defecto/s:

Nombre y apellidos. DNI.

E-mail.

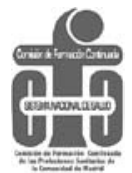




\section{Cuadro 3 (tomado de [1]).}

Un hombre de 60 años es trasladado a urgencias por la policía, al haberlo encontrado en estado inconsciente en la acera. Una vez determinado que la vía aérea está abierta el primer paso en el tratamiento debe ser la administración intravenosa de:

A. Examen del líquido cefalorraquídeo.

B. Glucosa con vitamina B, (tiamina).

C. Tomografía computarizada de la cabeza.

D. Fenitoína.

E. Diacepam.

Comentario: las opciones A y C no son coherentes y pierden valor como distractores.

- Proponer opciones mutuamente exclusivas u obvias.

- Incluir opciones que engloben otras opciones. Debe evitarse formular opciones globalizadoras porque supone dar pistas al examinando. En este sentido, no deben utilizarse como opciones las expresiones 'ninguna de las anteriores' o 'todas las anteriores' o 'las opciones A y B son correctas'. Cuando utilizamos la opción de 'ninguna de las anteriores', convertimos todas las opciones en preguntas de verdadero/falso y cada opción ha de ser evaluada. Por otra parte, cuando utilizamos estas expresiones hay una tendencia a que ésta sea la correcta (Cuadro 4).

\section{Cuadro 4 (tomado de [2]).}

En un esguince de tobillo, colocaremos un vendaje desde el muslo hacia el pie en

A.

B. ...

C....

D. Ninguna de las anteriores

E. Todas las anteriores

Comentario: no utilizar las expresiones de las opciones $\mathrm{D}$ y $\mathrm{E}_{\text {; }}$ en caso de utilizarlas, hacerlo en el orden correcto.

Generalmente es posible reemplazar 'ninguna de las anteriores' por un ítem que signifique aproximadamente lo mismo pero que sea más específico. Por ejemplo, en una pregunta que solicita al alumno que especifique 'el tratamiento farmacológico más adecuado', si se reemplaza 'ninguna de las anteriores' por 'en este momento no se deben administrar medi- camentos', se eliminará la ambigüedad de 'ninguna de las anteriores'.

Si utiliza 'todas las anteriores son ciertas' y todas las opciones son semejantes, se pierde efectividad.

- Usar términos imprecisos como 'posiblemente', 'está asociado a', 'es útil para', 'es importante', 'como puede' o 'podría ser,' 'normalmente' o 'con frecuencia,' 'nunca,' 'siempre' (Cuadro 5)... Cuando se utilizan los términos absolutos como 'nunca' o 'siempre' en las opciones, éstas suelen descartarse.

\section{Cuadro 5 (tomado de [1]).}

a) En pacientes que padecen demencia avanzada,

tipo Alzheimer, el defecto de memoria

A. Puede ser tratado correctamente con fosfatidilcolina (lecitina).

B. Podría constituir una secuela de enfermedad de Parkinson precoz.

C. Nunca se observa en pacientes con ovillos neurofibrilares en la autopsia.

D. Nunca es grave.

E. Posiblemente afecta al sistema colinérgico.

Comentario: eliminar 'puede,',podría,',nunca,',posiblemente'.

b) La obesidad mórbida en los primeros años de la adolescencia

A. Normalmente responde de manera drástica al régimen alimenticio.

B. A menudo se relaciona con trastornos endocrinos.

C. Tiene un $75 \%$ de posibilidades de desaparecer espontáneamente.

D. Tiene un mal pronóstico.

E. Normalmente responde a la farmacoterapia y a la psicoterapia intensiva.

Comentario: evitar los términos 'normalmente,' 'a menudo', etc.

- Formular opciones heterogéneas tanto en lo que se refiere a su longitud como a su complejidad. Habitualmente se tiende a que la opción más larga, más compleja o más completa sea la correcta (Cuadro 6).

- Repetir elementos en las opciones de tal manera que sean comunes a varias de ellas. Hay una tendencia a que la opción correcta sea la que contiene más elementos en común (Cuadros 6 y 7 ).

- Repetir palabras en las opciones que pueden estar en el enunciado (Cuadro 8). 


\section{Tabla I. Principales defectos técnicos a evitar.}

\section{No debemos hacer:}

1. Formular enunciados que supongan juicios de valor $u$ opiniones personales

2. Proponer opciones o distractores que no tengan correspondencia gramatical con el enunciado.

3. Proponer opciones mutuamente exclusivas u obvias tanto en sentido positivo como negativo.

4. Incluir opciones globalizadoras ('ninguna de las anteriores'o 'todas las anteriores')

5. Usar términos imprecisos como 'posiblemente,'está asociado a,'es útil para,'es importante,',como puede'o 'podría ser', 'normalmente' o 'con frecuencia,',nunca,',siempre,' 'mucho mayor','menor', etc.

6. Utilizar opciones heterogéneas tanto en lo que se refiere a su longitud como a su complejidad

7. Repetir elementos en las opciones de tal manera que sean comunes a varias

8. Repetir palabras en las opciones que pueden estar en el enunciado

9. Repetir palabras en las opciones en el enunciado y en las opciones que puedan facilitar pistas

10. Formular opciones complejas o dobles

11. No expresar correctamente o de forma homogénea los datos numéricos

12. Formular enunciados negativos ('cuál no es,',cuál es falsa,',todas son ciertas excepto...')

\section{Cuadro 6.}

En un paciente de 70 años diagnosticado de hiperaldosteronismo, hallará:

A. Hipernatremia e hiperkaliemia.

B. Hipernatremia e hipovolemia.

C. Hipernatremia e hipotensión.

D. Hipernatremia, hipokaliemia, hipervolemia e hipertensión.

E. Hipotensión.

Comentario: el hecho de que el término 'hipernatremia' esté en todas las opciones excepto en una hace que la opción en la que no está (opción E) pierda fuerza como distractor. La opción correcta (opción D) es la opción más completa y más larga.

\section{Cuadro 7 (tomado de [1]).}

Los anestésicos locales son más eficaces en su forma

A. Aniónica y actúan desde el interior de la membrana nerviosa.

B. Catiónica y actúan desde el interior de la membrana nerviosa.

C. Catiónica y actúan desde el exterior de la membrana nerviosa.

D. Sin carga y actúan desde el interior de la membrana nerviosa.

E. Sin carga y actúan desde el exterior de la membrana nerviosa.

Comentario: el término 'catiónico' aparece en dos de las opciones. La opción de que sea una forma con carga aparece en tres de las opciones. El término 'interior de la membrana' aparece en tres de las opciones. Hay muchas probabilidades de que la opción correcta sea la opción B.
Cuadro 8 (tomado de [2]).

En una historia clínica por crisis de 'angina de pecho', ¿qué dato le sugerirá un probable infarto de miocardio?:

A. Si la crisis apareció en reposo.

B. Si la crisis apareció durante un proceso febril.

C. Si la crisis apareció tras el esfuerzo.

D. Si la crisis apareció tras una emoción.

E. Si la crisis apareció conduciendo un vehículo.

Comentario: se debería redactar el enunciado como 'una crisis de angina de pecho le sugeriría un probable infarto de miocardio si ésta apareció':

A. En reposo.

B. Durante un proceso febril.

C. Tras el esfuerzo.

D. Tras una emoción

E. Conduciendo un vehículo.

- Repetir palabras en el enunciado y en las opciones que puedan facilitar pistas (Cuadro 9).

\section{Cuadro 9 (tomado de [1]).}

Un hombre de 58 años de edad, con antecedentes de alcoholismo e ingresos psiquiátricos previos presenta confusión y agitación. Comenta que siente que el mundo es algo 'irreal.' El síntoma se denomina:

A. Despersonalización.

B. Descarrilamiento.

C.'Desrealización'.

D. Déficit focal de memoria.

E. Ansiedad. 
Tabla II. Preguntas de respuesta múltiple (tomado de [1,2]).

1. La desaparición de la regla a partir de los 45-55 años se denomina:

A) Menopausia; B) Menarquia; C) Climaterio; D) Ninguna de las anteriores; E) Todas las anteriores

2. La anomalía congénita 'recto ciego' representa del total de las anomalías anorrectales: A) $67,5 \%$; B) $20 \%$; C) $3 \%$; D) $35 \%$; E) $50 \%$

3. La parotiditis tiene un período de incubación de:

A) 20-30 días; B) 10-15 días; C) 15-20 días; D) 30-40 días; E) 5-10 días

4. Con respecto a la diabetes es falso que:

A) No se producen alteraciones oculares; B) Es más frecuente en hombres que en mujeres; C) No produce alteraciones hepáticas; D) Ninguna de las anteriores; E) Todas las anteriores

5. El prurito en pacientes que padecen enfermedad de Hodgkin es:

A) Frecuente; B) Poco frecuente; C) Raro; D) Habitual

6. Entre los siguientes microorganismos, el que provoca la aparición de endocarditis bacteriana de forma aguda es: A) Streptococcus viridans; B) Staphylococcus aureus; C) Neumococo; D) Haemophilus influenzae; E) Estreptococo $\beta$-hemolítico

7 Con respecto a los glucocorticoides, es falso que:

A) Aumente la glicólisis; B) No aumente la neoglucogénesis; C) Estimule la síntesis proteica; D) Todas las anteriores son falsas; E) Ninguna de las anteriores es cierta

8. ¿En qué momento considera usted que se presenta temblor en la enfermedad de Wilson?

A) Durante el sueño; B) Durante el reposo; C) En posición erecta; D) Durante los movimientos coordinados, en reposo y al mantener la postura; E) Ninguna de las anteriores

\section{Respecto al nistagmo, todas las siguientes opciones son correctas excepto:}

A) El nistagmo vestibular se caracteriza por un movimiento lateral rápido de los ojos hacia el lado contrario de la rotación de la cabeza, seguido de otro lento hacia el mismo lado de la rotación; B) Puede aparecer nistagmo en una persona cuando observa un objeto que se mueve; C) Puede producirse nistagmo por activación térmica del aparato vestibular; D) Puede producirse nistagmo por rotación de la cabeza; E) El nistagmo provocado es un hecho fisiológico.

10. Un hombre sano de 33 años de edad tiene debilidad leve y episodios ocasionales de dolor abdominal intenso y continuo con algunos calambres, pero sin diarrea. Una tía y un primo han padecido episodios similares. Durante una crisis, su abdomen se distiende y disminuyen los ruidos intestinales. El examen neurológico muestra debilidad leve en la parte superior de los brazos. Estos datos sugieren que existe una anomalía en la vía biosintética:

A) Del colágeno; B) De los corticosteroides; C) De los ácidos grasos; D) De la glucosa; E) Del hemo

- Formular opciones complejas o dobles (p. ej., ¿cuál es el diagnóstico y el tratamiento adecuados?'). En estos casos, la situación empeora si alguna o algunas de las opciones no contienen ambos aspectos. Se debe evitar también formular las preguntas con enunciados del tipo 'cuál' o 'cuáles'. Si se formula así y alguna opción contiene más de un elemento, tiene más fuerza para ser la correcta.

- Expresar incorrectamente y de forma no homogénea los datos numéricos. Éstos deben expresarse de forma homogénea, en orden creciente o decreciente, con el mismo grado de detalle, haciendo constar las unidades si procede. Si se expresan intervalos, todas las opciones deben expresar intervalos, y dichos intervalos no deben solaparse. Unas cifras no pueden englobar otras (sobre todo cuando las opciones indican mayor o menor que...). Si no utiliza el mismo patrón en todas las cifras, la respuesta correcta suele corresponderse con la más específica.

- Formular enunciados con preguntas del tipo ¿cuál es la conducta, la terapéutica incorrecta?', ‘¿cuál de las opciones es falsa?.' En estos ca- 
sos, el alumno puede saber la incorrecta, pero nadie nos asegura que conozca la correcta. Formular enunciados del tipo 'las opciones a continuación son correctas excepto....

- Utilizar proposiciones negativas o dobles negativas, ni incluir respuestas negativas cuando el enunciado es también negativo (p. ej., 'no es cierto que en la mononucleosis infecciosa no se da...' o 'respecto al ECG es falso que a)..., b)... c)..., d..., e) Ninguna de las anteriores'.

El lector hallará en la tabla II diez PRM en las que deberá identificar los posibles defectos técnicos que contengan y, de acuerdo con ello, cumplimentar el test de evaluación correspondiente.

\section{Bibliografía}

1. Case SM, Swanson DB. National Board of Medical Examiners. Cómo elaborar preguntas para evaluaciones escritas en el área de ciencias básicas y clínicas. 2006. URL: http://www.nbme.org/pdf/iwg-sp/iwg-spanish2006-app. pdf. [10.07.2010].

2. García-Barbero M, Castejón-Ortega JV, Cancillo-Salas J, Sánchez-Pérez SP. Como confeccionar preguntas test y prac. Alicante: División de Educación Médica, ICE, Universidad de Alicante; 1987. 